\title{
Penetrating ulcer of the aortic arch presenting with hemoptysis
}

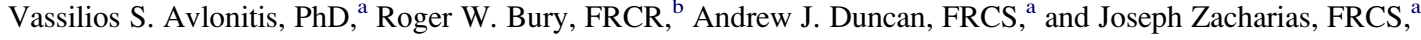 \\ Blackpool, United Kingdom
}

Penetrating aortic ulcers (PAUs) are increasingly recognized as a separate entity. We report a PAU with unusual presentation and discuss difficulties in diagnosis and management.

\section{CLINICAL SUMMARY}

A 58-year-old nonsmoker presented with a 6-month history of hemoptysis and a 1-month history of left-sided chest pain. He was known to have poorly controlled hypertension. Physical examination was unremarkable. Chest radiographic analysis showed a lesion in the left lung apex lying against the aortic arch. Contrast computed tomographic (CT) scanning demonstrated continuity between the lesion and the aortic lumen. Positron emission tomographic analysis confirmed high uptake in the lesion, and the patient was referred to us as having a case of lung cancer with extension into the aorta (Figure 1). However, multislice CT analysis with 3-dimensional reconstruction clarified the presence of a PAU in the arch leaking into the lung, with surrounding hematoma (Figure 2). The patient continued to experience pain, and we proceeded to surgical intervention.

Hypothermic $\left(24^{\circ} \mathrm{C}\right)$ cardiopulmonary bypass was established between the right atrium and the right subclavian artery. Myocardial protection was with intermittent antegrade cold blood cardioplegia. The ulcer was full of clot and penetrating into the lung. The clot was sent for culture. The base of the ulcer extended to the descending aorta, adhering to the left lung and the phrenic nerve. Dissection to free the descending aorta was impossible. The aortic wall was thickened, inflamed, and friable. The ulcer was excised, and the aortic defect was patched with Dacron. Because of the poor quality of aortic tissue, the bleeding from the suture line could not be controlled, and therefore the arch was replaced with a Dacron (Golscal, Sulzer Vascutek, UK) graft, leaving an elephant trunk in the descending aorta. An island of aorta containing the innominate and left common carotid arteries was reimplanted onto the arch. The patient was weaned off cardio-

From the Departments of Cardiothoracic Surgery ${ }^{\mathrm{a}}$ and Radiology, ${ }^{\mathrm{b}}$ Lancashire Cardiac Centre, Blackpool, United Kingdom.

Received for publication March 11, 2008; accepted for publication March 23, 2008. Address for reprints: Vassilios S. Avlonitis, PhD, Level 9, Plateau Building, Derriford Hospital, Derriford Rd, Plymouth, PL6 8DH, United Kingdom (E-mail: avlonitis@ hotmail.com).

J Thorac Cardiovasc Surg 2009;137:e10-2

$0022-5223 / \$ 36.00$

Copyright (c) 2009 by The American Association for Thoracic Surgery doi:10.1016/j.jtcvs.2008.03.031 pulmonary bypass (time, 372 minutes) without inotropes. Because of ongoing coagulopathy, hemostasis was impossible to achieve, despite blood product transfusion. The bleeding only stopped after administration of Factor VIIa.

The patient was extubated within 24 hours and discharged from the intensive care unit. The clot culture grew Staphylococcus aureus. He was started on flucloxacillin for 4 weeks and discharged on day 11 . He was readmitted 2 months later with malaise, rigors, fever, and a C-reactive protein level of $80 \mathrm{mg} / \mathrm{L}$. CT scanning showed soft tissue thickening around the graft, resolution of the lung hematoma, and no fluid collection. The results of blood cultures were negative. Based on the previous history, he was started on intravenous flucloxacillin, rifampicin, and gentamicin for 4 weeks. He responded well and was discharged. One year later, he remains well.

\section{DISCUSSION}

PAUs result from atheroma, ulcerating and disrupting the internal elastic lamina, burrowing through all aortic layers. ${ }^{1}$ They can remain quiescent or lead to dissection, intramural hematoma, aneurysm or pseudoaneurysm formation, fistulation into adjacent organs, or free wall rupture. ${ }^{2}$ The most common presentation is with anterior chest pain or intrascapular pain caused by dissection or intramural hematoma. ${ }^{3}$ Hemoptysis is an extremely rare manifestation. We could only find one case presenting with hemoptysis in the literature. ${ }^{4}$ That case was managed with hypertension control, and the pulmonary hematoma gradually disappeared. In our case, because of persistent pain, we proceeded to surgical intervention. We did not perform a pulmonary resection, expecting the lung hematoma to resolve after aortic repair.

The ulcer penetrating into the lung raises a diagnostic dilemma and can be mistaken for lung cancer invading the aorta. Positron emission tomographic scanning showing activity in the lung suggested the latter. Multislice CT scanning was more accurate in delineating the exact pathology.

At surgical intervention, there was loss of tissue planes between the lung and aortic arch caused by hematoma and inflammation. The thickening of the aortic intima made construction of a hemostatic suture line impossible when patching was attempted. Therefore we excluded the abnormal area with an elephant trunk procedure. Ongoing coagulopathy obliged us to use Factor VII to achieve hemostasis, with impressive results. There are increasing reports of its successful use in cardiac surgery, and we would recommend its use in 

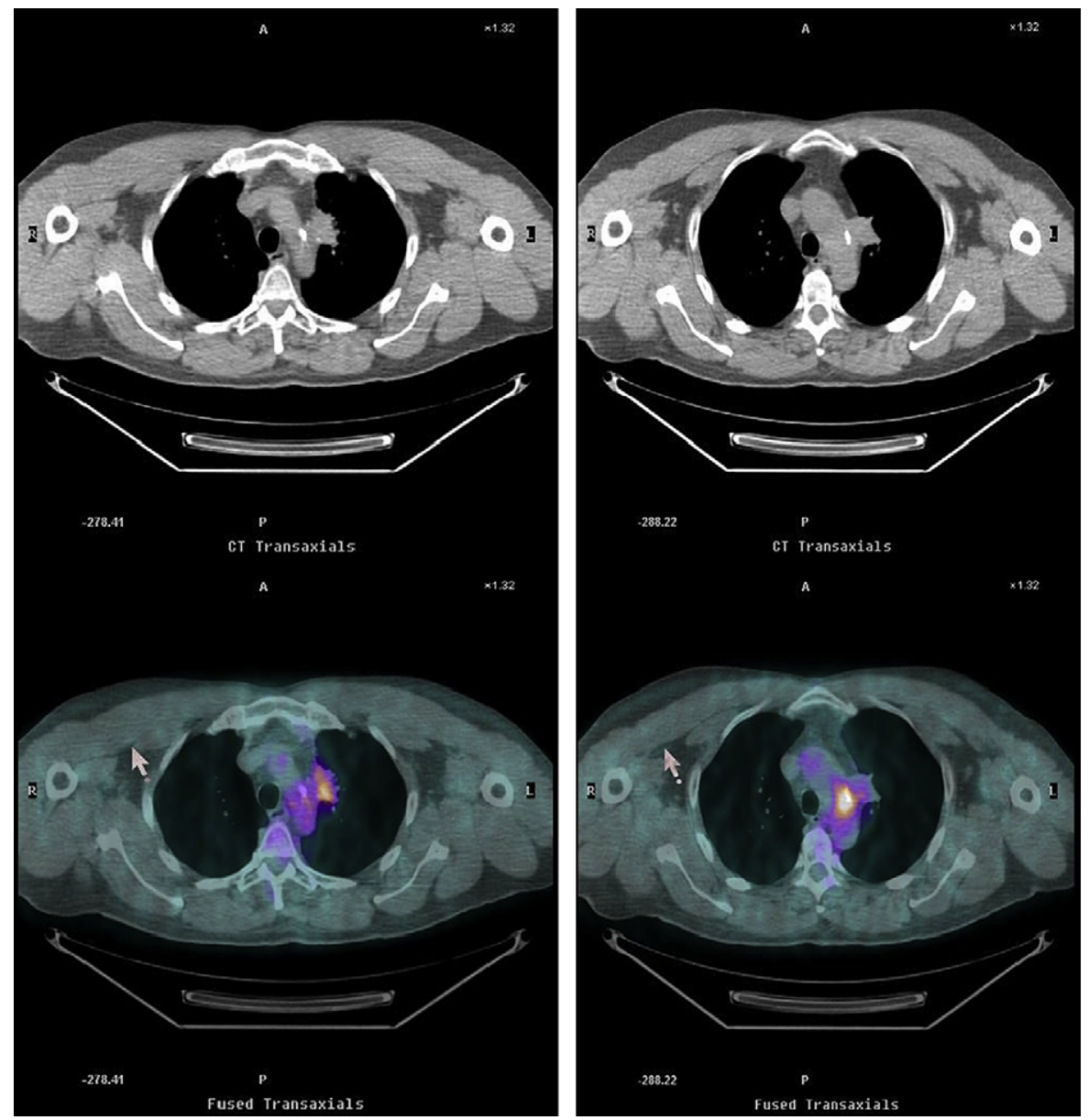

FIGURE 1. Positron emission tomogram demonstrating high uptake in a left upper lobe lung lesion extending into the aortic arch. The lesion was thought to be lung cancer with aortic invasion.

aortic surgery cases with coagulopathy unresponsive to standard blood product transfusion. ${ }^{5}$

The postoperative course of this patient was uneventful, with the exception of the graft infection. Contamination of the area with lung pathogens was inevitable. In such cases proactive and aggressive management is recommended.
Specimens for microbiologic cultures should be obtained intraoperatively, and a prolonged course of antibiotics might be required.

In conclusion, PAUs involving the lung can be mistaken for lung malignancy invading the aorta. Surgical management can be technically challenging, and the possibility of
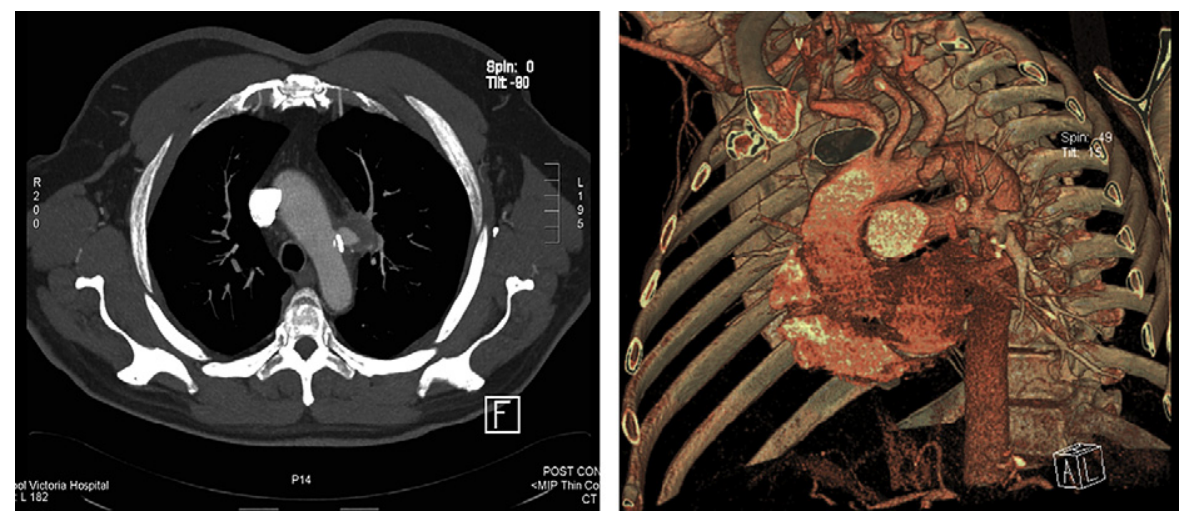

FIGURE 2. Computed tomographic and 3-dimensional reconstruction showing an aortic ulcer penetrating into the lung. 
postoperative graft infection must be considered and managed aggressively.

\footnotetext{
References

1. Stanson AW, Kazmier FJ, Hollier LH, Edwards WD, Pairolero PC, Sheedy PF, et al. Penetrating atherosclerotic ulcers of the thoracic aorta: natural history and
} clinicopathologic correlations. Ann Vasc Surg. 1986;1:15-23.
2. Troxler M, Mavor AI, Homer-Vanniasinkam S. Penetrating atherosclerotic ulcers of the aorta. Br J Surg. 2001;88:1169-77.

3. Coady MA, Rizzo JA, Hammond GL, Pierce JG, Kopf GS, Elefteriades JA. Penetrating ulcer of the thoracic aorta: What is it? How do we recognize it? How do we manage it? J Vasc Surg. 1998;27:1006-16.

4. Fukushima M, Seino Y, Yoshikawa M, Ueda Y, Takano T. A case of penetrating aortic atherosclerotic ulcer with hemoptysis. Jpn Heart J. 2000;41:781-5.

5. Raivio P, Suojaranta-Ylinen R, Kuitunen AH. Recombinant factor VIIa in the treatment of postoperative hemorrhage after cardiac surgery. Ann Thorac Surg. 2005; 80:66-71.

\title{
Concomitant cardiac and cerebral leiomyosarcoma: A challenge for surgical and adjuvant therapy
}

\author{
Christoph A. Tschan, MD, ${ }^{\mathrm{a}}$ M. Javad Mirzayan, MD, ${ }^{\mathrm{a}}$ Alexandru C. Stan, MD, ${ }^{\mathrm{b}}$ Maximilian A. Pichlmaier, MD, ${ }^{\mathrm{c}}$ and \\ Joachim K. Krauss, MD, ${ }^{a}$ Hannover, Germany
}

Primary sarcomas of the heart are rare, ${ }^{1}$ and features of leiomyosarcoma are present in only $1 \%$ to $9 \% .^{2}$ Although cardiac tumors, such as myxomas, occasionally can metastasize to the brain, this scenario is uncommon for leiomyosarcoma. Only 3 patients with leiomyosarcoma of the heart metastasizing to the brain have been reported within the past 40 years. $^{3-5}$ Here we report on a patient with the concomitant occurrence of a cerebral leiomyosarcoma and a cardiac leiomyosarcoma presenting initially with a generalized seizure. This is the first description of a patient in whom a cardiac leiomyosarcoma was detected only after diagnosis of the cerebral tumor.

\section{CLINICAL SUMMARY}

A 35-year-old man presented with a generalized seizure. A cranial magnetic resonance scan showed a large cystic right frontal tumor (Figure 1, $A$ and $B$ ). Diagnostic work-up raised the suspicion of a cardiac tumor. Magnetic resonance scans of the chest (Figure 1, $C$ and $D$ ) revealed an intracardiac neoplasm. Coronary angiographic and electrocardiographic analyses were without pathologic findings. Computed tomographic scanning of the whole body did not reveal any other tumor. First, the cardiac tumor was resected under extracorporeal circulation through a median sternotomy. The left

From the Department of Neurosurgery, ${ }^{\mathrm{a}}$ Institute of Pathology, ${ }^{\mathrm{b}}$ and Department of Cardiac, Thoracic, Transplantation and Vascular Surgery, ${ }^{c}$ Hannover Medical School, Hannover, Germany.

Received for publication April 8, 2008; revisions received May 8, 2008; accepted for publication May 21, 2008.

Address for reprints: Joachim K. Krauss, MD, Department of Neurosurgery, Hannover Medical School, Carl-Neuberg-Str. 1, D-30625 Hannover, Germany (E-mail: Krauss.Joachim@mh-hannover.de).

J Thorac Cardiovasc Surg 2009;137:e12-4

$0022-5223 / \$ 36.00$

Copyright (c) 2009 by The American Association for Thoracic Surgery doi:10.1016/j.jtcvs.2008.05.031 atrium was reconstructed with autogenic pericardium. Three weeks later, the frontal tumor was removed.

Both resected tumor specimens displayed characteristic histomorphologic features of a poorly differentiated leiomyosarcoma (Figure 1, $E$ and $F$ ). The immunohistochemical profile of the cardiac tumor was positive for $\alpha$-actin, smooth muscle actin, bcl-2, S-100, and CD117, with a Ki-67/MIB 1 proliferation index of $30 \%$. The cerebral tumor was positive for CD117 and vimentin, and a Ki-67/MIB 1 proliferation index of $40 \%$ was found.

Radiation therapy at 46 Gy was administered to the right frontal lobe. At 6 months of follow-up, imaging studies did not reveal residual tumors. Three months later, however, cerebral tumor recurrence was detected. The patient underwent a second craniotomy. Subsequent tumor staging revealed a spleen metastasis, followed by splenectomy. The tumor specimen demonstrated a capsular breakthrough and progression of dedifferentiation in terms of lack of expression of characteristic immunohistochemical markers.

Three months thereafter, another tumor recurrence occurred in the right frontal lobe. Whole-body staging showed widespread intra-abdominal metastases. A third craniotomy was performed. Chemotherapy was administered with doxorubicin and ifosfamide. Despite chemotherapy, the intracranial tumor recurred again. Therefore another course of radiation therapy was administered.

During adjuvant chemotherapy and radiotherapy, the frontal tumor progressed. With regard to the patient's good clinical condition, the decision was made to perform another craniotomy. Tumor staging at this time showed exploding intra-abdominal metastases. During the further course, catheter-induced sepsis developed. Laboratory examinations excluded HIV infection and other immunodeficiency syndromes. The patient died of septicemia 18 months after the primary diagnosis. 